\title{
Uniaxial Tensile Load of Lattice Structures Produced by Metal Additive Manufacturing
}

Pavel Hanzl, Ivana Zetková, Milan Daňa

Faculty of Mechanical Engineering, University of West Bohemia. Univerzitní 2732/8, 30614 Pilsen. Czech Republic. E-mail: hanzlp@rti.zcu.cz, zetkova@rti.zcu.cz,danam@rti.zcu.cz

\begin{abstract}
Lightweight constructions are in demand for applications which require low weight accompanied by rigidity. From this perspective, lattice structures are an interesting design solution in conjunction with additive manufacturing technology. This study addresses promising lattice topologies. A range of metallic lattice structures was produced using Direct Metal Laser Sintering technology and the samples were exposed to uniaxial tensile load. During the tensile tests crack initiations and locations of the breaks were observed. The results clarify the weaknesses of the cellular lattices and reveal their maximum tensile load. First steps have been taken to meet the demand for optimized lightweight AM products.
\end{abstract}

Keywords: Lattice Structures, Rigid Constructions, Additive Manufacturing, Selective Laser Melting, Direct Metal Laser Sintering

\section{Introduction}

Requirements for energy efficient machines are constantly increasing. For example, research and development in the automotive industry is making efforts towards weight reduction to help reduce fuel consumption and exhaust emissions. This can be achieved by either substituting current materials with higher grade materials, or design optimization. The first approach is usually accompanied by worse machinability because these materials have high specific strength. These materials are titanium alloys, high strength steels and aluminium/magnesium. The second approach is based on adapting the structural design to the loading conditions. Examples of this strategy are sandwich design, which is designed to give high rigidity accompanied by low weight. This strategy places high demands on manufacturing technologies due to the high complexity of the parts. [1]

Porous structures can have either a non-stochastic or stochastic character. Metal foams belong to the first group of non-stochastic structures. They are characterized by irregular distribution of cavities. Therefore, their geometry is not precisely defined. Metal foams offer a wide range of attributes including low thermal conductivity, electromagnetic wave shielding and great toughness. Lattice cellular structures, which belong to the second group, have similar properties. These structures are formed from struts and their geometry is regular. Various methods exist for producing these regular structures. [2]

Metal additive manufacturing (MAM) has the ability to produce highly complex parts because parts are built layer by layer. Numerous scientific publications have verified its suitability for producing complex cellular lattice structures. [3] Technology based on melting of a powder bed includes Direct Metal Laser Sintering, which was used for the experiments in this paper.

Metal parts made by this manufacturing technology usually have a fine structure. Therefore, they typically achieve better mechanical properties than cast materials. They may even have properties comparable with forming steel. The finer structure is the result of high cooling rates in the locally melted area of the thin metal layer. This can lead to non-equilibrium phase and generation of internal stress. [4]

Nevertheless, this technology has its limits due to the method of melting in the powder bed. One of the downsides is the surface quality. A 'staircase' effect is evident on the molten surface of the parts. Additionally, overhangs usually require supports to hold the critical areas during the process. These support structures are built together with the components, help prevent distortion from thermal stresses and anchor the overhanging area to the building platform. The support structures are removed after the production is complete. [4]

Recent studies in the field of AM have investigated the mechanical strength properties of cellular lattice structures. The majority of the studies focus on pressure tests of porous structures. Nevertheless, paper [1] investigated the tensile behaviour of porous samples. Finite Element Analysis (FEA) is often included in the studies.

The topology and arrangement of the basic cell unit of a non-stochastic structure is an important aspect for increasing the specific load capacity. The ultimate resistance to damage results from the geometry and the loading situation. Therefore, multiple parameters are involved in the process of optimization of parts. Finite Element Analysis is an appropriate method for doing this. This study investigates the maximum load capacities of different topologies by observing crack initiations and the locations of the breaks.

\section{Experimental details}

The experiment comprises seven topologies with selfsupporting unit cells, as shown in Figure 1. All of the samples have a $5 \mathrm{~mm}$ unit size and the volume fraction is set at $13.5 \%$. The dimension of the porous core is $25 \mathrm{~mm}$ Cubic units such as the Body Centred Cubic (BCC) and Face Centred Cubic (FCC) form the basis for the other variations such as $\mathrm{PFCC}$ and $\mathrm{BCCz}$, where struts are added to gain stiffness along the $\mathrm{z}$ axis. $\mathrm{F}_{2} \mathrm{BCC}$ is created by combining BCC and FCC units.

Topologies, except BCC, Gyroid, Rhombic, do not have identical characteristics along all the main axes. 
Strength characteristics in directions $x$ and $y$ are fully interchangeable. Therefore, these topologies are tested in two load directions $x$ and $z$. The samples were manufactured so that their main axes are in the horizontal position.
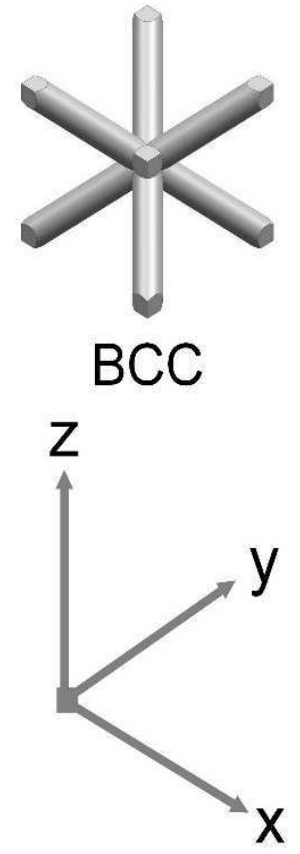
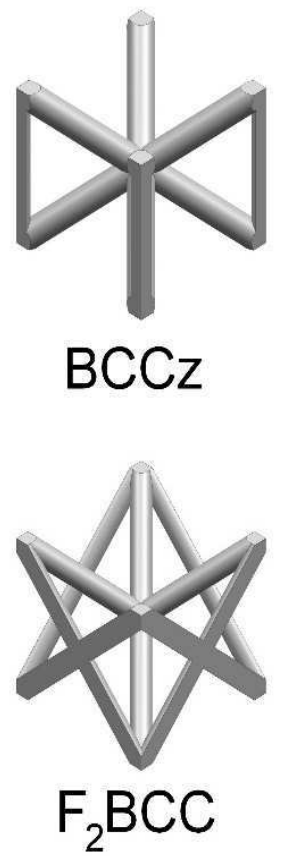
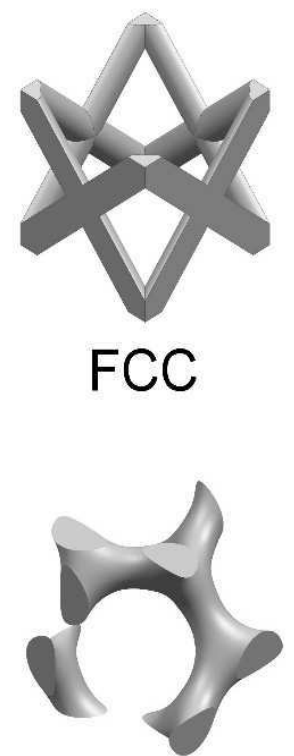

Gyroid
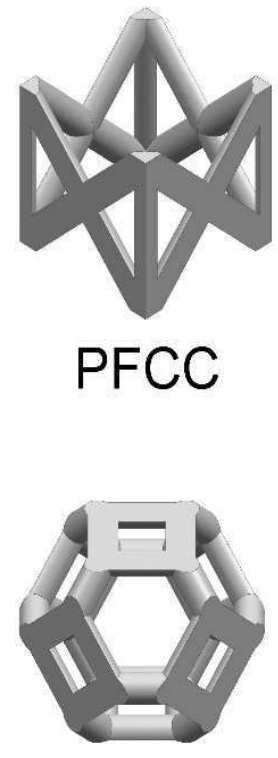

Rhombic

Fig. 1 Unit cell topologies

Tensile samples are produced by DMLS and their design is adapted for rigid clamping to deform only the porous core of the sample. Figure 2 shows the gyroid open cell geometry with dimensions of $25 \mathrm{~mm} \times 25 \mathrm{~mm} \times 25$ mm. Standard 'EOS_DirectTool' process parameters were used for material 1.2709 according to European classification.

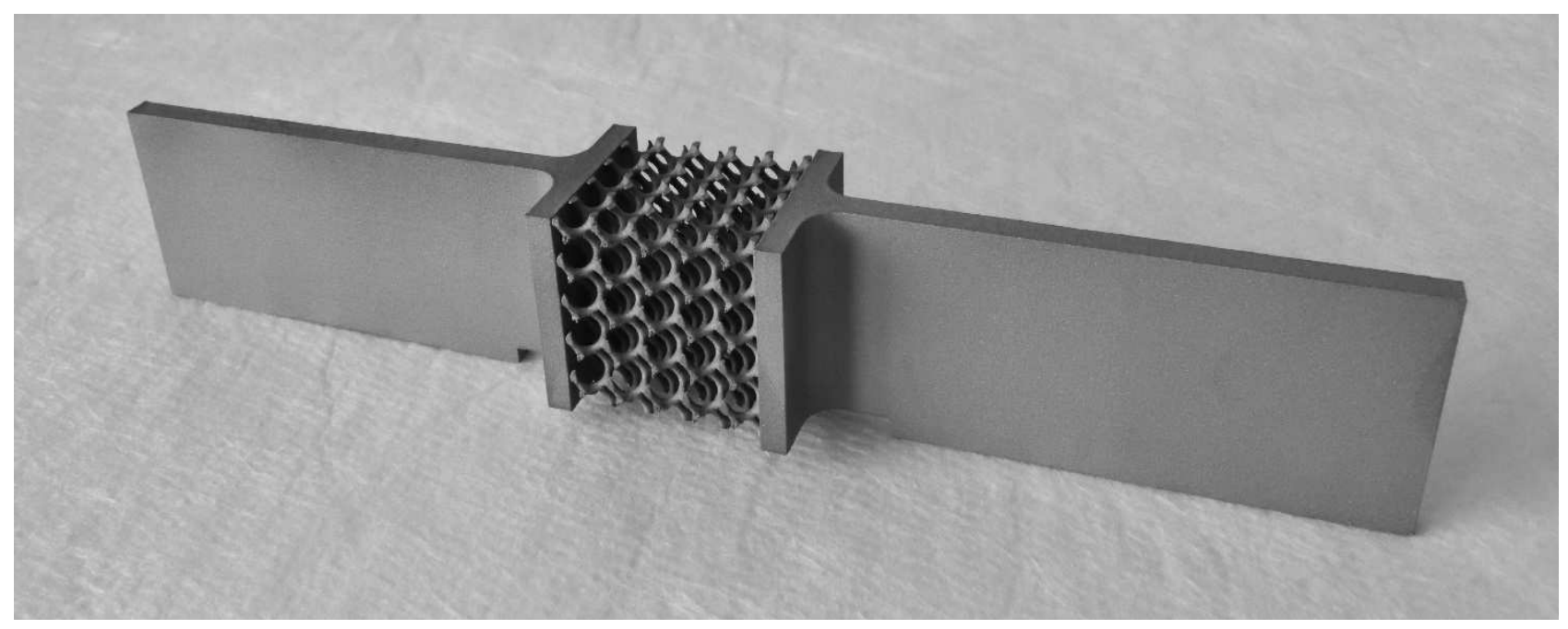

Fig. 2 Tensile sample with gyroid topology

\section{Experiment}

Tensile tests are often used to determine material properties. When a porous structure is tested, the test evaluates not only the material but also the geometry of the structure. The proof of this fact is load capacity, which is different for each topology. The yield strength always occurs when the material is permanently deformed and ultimate strength is the limit stress that leads to tensile failure. Therefore, this experiment does not evaluate material properties, but properties of topologies in connection with the 1.2709 material.

The results are shown as force loading. Fmax is the force acting on the cross-sectional area $25 \mathrm{~mm} \times 25 \mathrm{~mm}$, that causes sample destruction. Fp0.2 is the force equivalent to the yield strength resistance to permanent elongation of $0.2 \%$. Figure 3 shows the values of the tensile tests. 

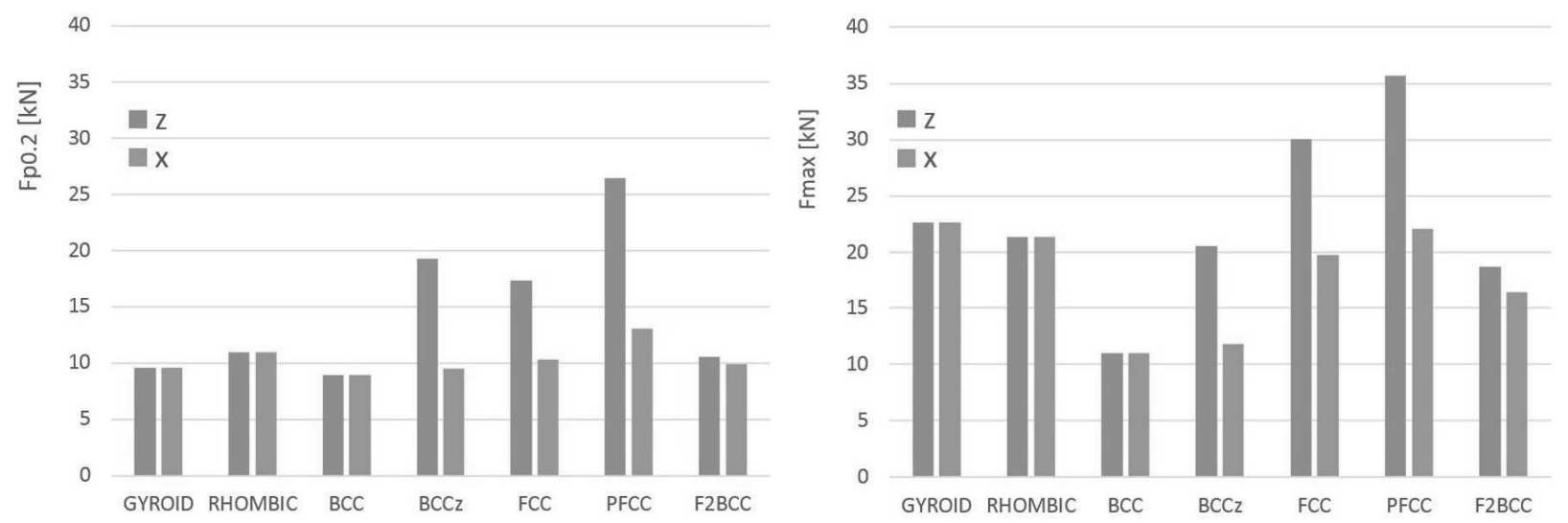

Fig. 3 The load capacities of the lattice structures in the experiment

All the samples except Gyroid and BCC were tested along directions $\underline{x}$ and $\underline{z}$ because these topologies usually have higher stiffness in the direction of the $\underline{z}$ axis. The PFCC topology reached the highest load capacity without permanent deformations along both directions. It also had the least balanced results. However, this geometry is pre- ferred in applications where the highest stiffness is required for a uniaxial load. FCC and BCCz provided interesting results in this case. The deformation curves did not show a significant yield strength. The load force increased until an interruption of cohesion from some struts was created.

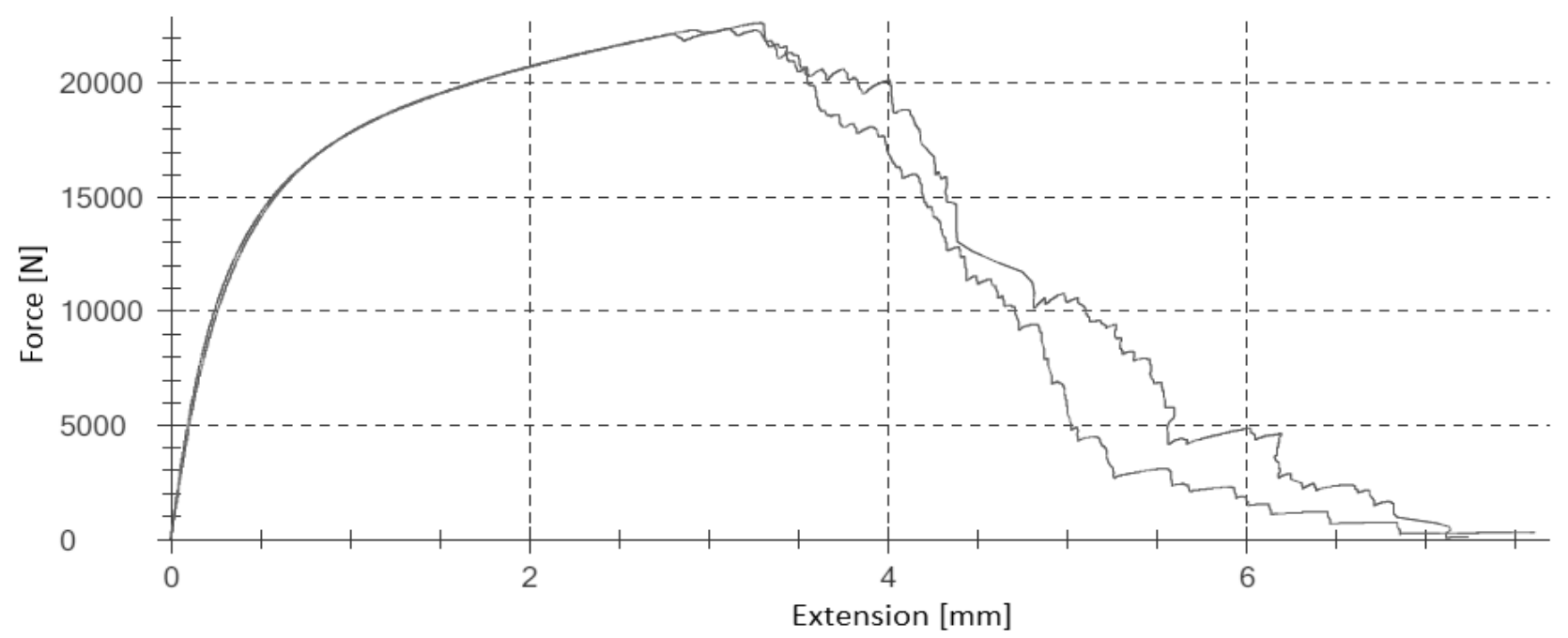

When the maximum loads were reached, the samples were broken. In most cases, the breach of the sample was gradual except for the PFCC topology. These topologies loaded along the $\underline{z}$ axis were broken abruptly. Reinforcement of the struts in the connection with the rigid plates should increase the maximum load. The crack initiations were always on the surface of the porous core and then the crack spread throughout the body. The samples with topologies BCC, FCC, PFCC and F2BCC cracked at the connection of the porous core with the rigid plate. The Rhombic and $\mathrm{BCCz}$ topologies cracked at an angle of $45^{\circ}$. Only the gyroid structure broke in the centre of the sample. a)

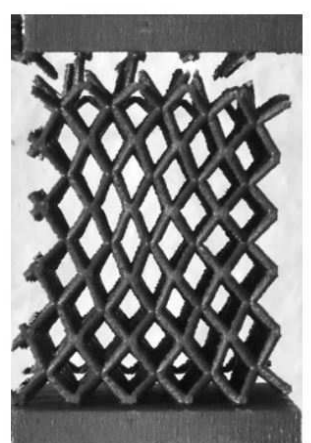

b)

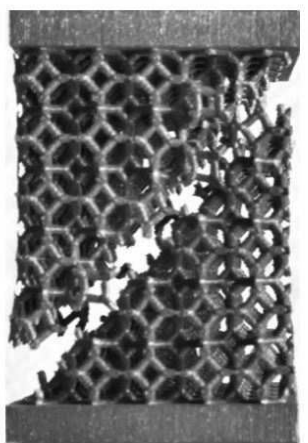

c)

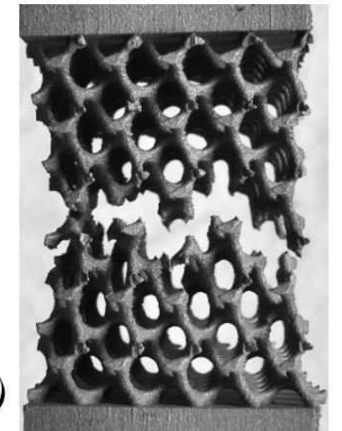

Fig. 4 Crack propagation: a) BCC, FCC, PFCC and $F_{2} B C C$; b) Rhombic and BCCz; c) Gyroid 


\section{Conclusion}

Lattice structures are used for topological optimization because of their properties. This article is focused on the maximum load capacity of uniaxial tension. The experiment tests different kinds of porous topologies. The range of load capacities were found to be from $19 \mathrm{kN}$ to $40 \mathrm{kN}$. This shows that there is a great difference between topologies of samples with the same weight and dimensions.

Theory about stiffness of lattice structures is following. If stretch-dominated strain prevails in the porous core, the lattice structure has an excellent stiffness to weight ratio. In the opposite case, a blend-dominated character of topology is preferable for absorption of mechanical energy and vibration. This is based on the Maxwell rule. More can be read in papers by V. S. Deshpande et al. [6] and [7].

The results proved the highest load capacity to be in the PFCC topology. This geometry combines diagonal and vertical struts along direction $\underline{z}$. This distribution is the most effective of the tested porous samples. It means that tension in the porous core is of a stretch-dominated character. The Gyroid and BCC topologies also achieve great strength.

Gyroid topology can be very useful in applications requiring high stiffness and the ability to absorb vibration. Its geometry is described by an implicit mathematical equation, unlike the other structures in the experiment. Therefore, the Gyroid has its own specific properties and further research will be addressed to them.

The crack initiations of specimens were always on the surface of the porous core and then the crack spread throughout the body. Variable cross-sections of the struts in relation to local specimen failure can increase the maximum load capacity.

Tensile tests are often used to determine material properties. When testing a porous structure, the test evaluates not only the material but also the geometry of the structure. The proof of this fact is that the load capacity is different for each topology. The yield strength always occurs when the material is permanently deformed and ultimate strength is the limit stress that leads to tensile failure.

This paper confirms that lattice structures are interesting for the topological optimization of parts. The purpose of these modifications to component design is to reduce weight while maintaining strength requirements. The next goal of the research will be to find a way to apply the benefits of different kinds of lattice structures in mechanical engineering.

\section{Acknowledgement}

This paper is based upon work sponsored by project SGS-2016-005.

\section{References}

[1] BRENNE, F., NIENDORF, T., MAIER, H.J. (2013) Additively manufactured cellular structures: Impact of microstructure and local strains on the monotonic and cyclic behaviour under uniaxial and bending load, pp. 1558-1564. NOVAK, J., NOVOTNY, P., NOVY, K. (1990). Sample Book, pp. $58-68$, Vo. 213, Issue 9, Journal of Materials Processing Technology, ISSN 0924-0136

[2] SEDLAK, J., KUDLACOVA, B., ZEMCIK, O., JAROS, A., SLANY, M. (2017) Production of Planetary Mechanism Mechanism Model Prototype using Additive Method of Rapid Prototyping, Published by Manufacturing Technology, Vol. 17, No. 3, pp. 374-381, ISSN 1213-2489

[3] HANZL, P., ZETKOVÁ, I., DAŇA, M. (2017) Effect of Selected Factors on the Accuracy of Load Capacity of the Schoen Gyroid Cellular Structure, Published by Manufacturing Technology, Vol. 17, No. 3, pp. 302-306, ISSN 12132489

[4] KUČEROVÁ, L., ZETKOVÁ, I. (2016) Metallography of 3D printed 1.2709 tool steel. Published by Manufacturing Technology, Vol. 16, No. 1, pp. 140-144, ISSN 1213-2489

[5] HUSSEIN, A., HAO, L., YAN, C., EVERSON, R., YOUNG, P. (2013) Advanced lattice support structures for metal additive manufacturing, $\mathrm{Pu}-$ blished by Elsevier Ltd, Journal of Materials Processing Technology 213, pp. 1019-1026

[6] DESHPANDE, V.S., ASHBY, M.F., FLECK, N.A. (2001) Foam topology: bending versus stretching dominated architectures, Acta Materialia, Volume 49, Issue 6, Pages 1035-1040, ISSN $1359-6454$

[7] DESHPANDE, V.S., ASHBY, M.F., FLECK, N.A. (2001) Effective properties of the octet-truss lattice material, Journal of the Mechanics and Physics of Solids 49, Pages 1747-1769, PII: S0022-5069(01)00010-2 